\title{
Effective Length Of Quantum Confinement And Polaron Effect In A GaAs Film Deposited On $\mathrm{Al}_{0.3} \mathrm{Ga}_{0.7}$ As Substrate
}

\author{
Bing-Can Liu ${ }^{1, \mathrm{a}}$, Yong-Feng $\mathrm{He}^{1, \mathrm{~b}}$, Qiang Tian ${ }^{2, \mathrm{c}}$ \\ ${ }^{1}$ Department of Fundamental Courses, Academy of Armored Force Engineering, Beijing 100072, \\ China \\ ${ }^{2}$ Department of Physics, Beijing Normal University, Beijing 100875, China \\ ayoiobnu@sina.com, bheyf2014@126.com, 'tianqiangbnu@163.com
}

Keywords: Fractional-dimensional approach; Polaron effect; GaAs film

\begin{abstract}
The polaron confined in a $\mathrm{GaAs}$ film deposited on $\mathrm{Al}_{0.3} \mathrm{Ga}_{0.7} \mathrm{As}$ substrate are investigated by using the framework of the fractional-dimensional space approach. The numerical results for the polaron binding energy and effective mass as functions of the film thickness for different substrate thickness are obtained. It is shown that the polaron binding energy as a function of the film thickness for different values of substrate thickness has complicated structure for the narrow and medium film thickness.
\end{abstract}

\section{Introduction}

The fractional dimensional space approach, to our knowledge, was first applied by He [1,2], to study optical properties of anisotropic materials. In this approach the anisotropic interactions in real 3D space are treated as isotropic ones in an effective fractional-dimensional environment the dimension of which constitutes a measure of the degree of anisotropy of the actual physical system. The main advantage of this approach lies in the fact that all information about a perturbation can be introduced in a single value-the dimensionality. Thus given this simple value, the real system can be modeled in a simple analytical way. A simple analytical method has been developed by Mathieu et al. [3] to calculate the exciton binding energies in single quantum well structures by using the model of fractional dimensional space. This model can also be used to analyze excitonic features in semiconductor quantum wells [4,5]. Further, it can be adapted for magnetic-field-induced effects on shallow-donor states [6,7], biexciton [8,9], impurity states [10-12] and the Stark shift of excitonic complexes [13] in semiconductor heterostructures.

However, most of previous works have mainly been focused on quantum well structures and only a few works deal with film structures [14,15]. In this paper, we extend the fractional dimensional space approach to the case of a polaron confined to a GaAs film deposited on the $\mathrm{Al}_{0.3} \mathrm{Ga}_{0.7} \mathrm{As}$ substrate structure by using a set of material parameters as discussed by Smondyrev et al. [16]. In order to obtain the value of the fractional dimension $D$, the main task is to determine the length of confinement in the film structures; however, the length of confinement defined in previous works is not adaptive in film structures. In this paper, we further calculated the polaron binding energy and effective mass in film structures based on our previous work [17]. 

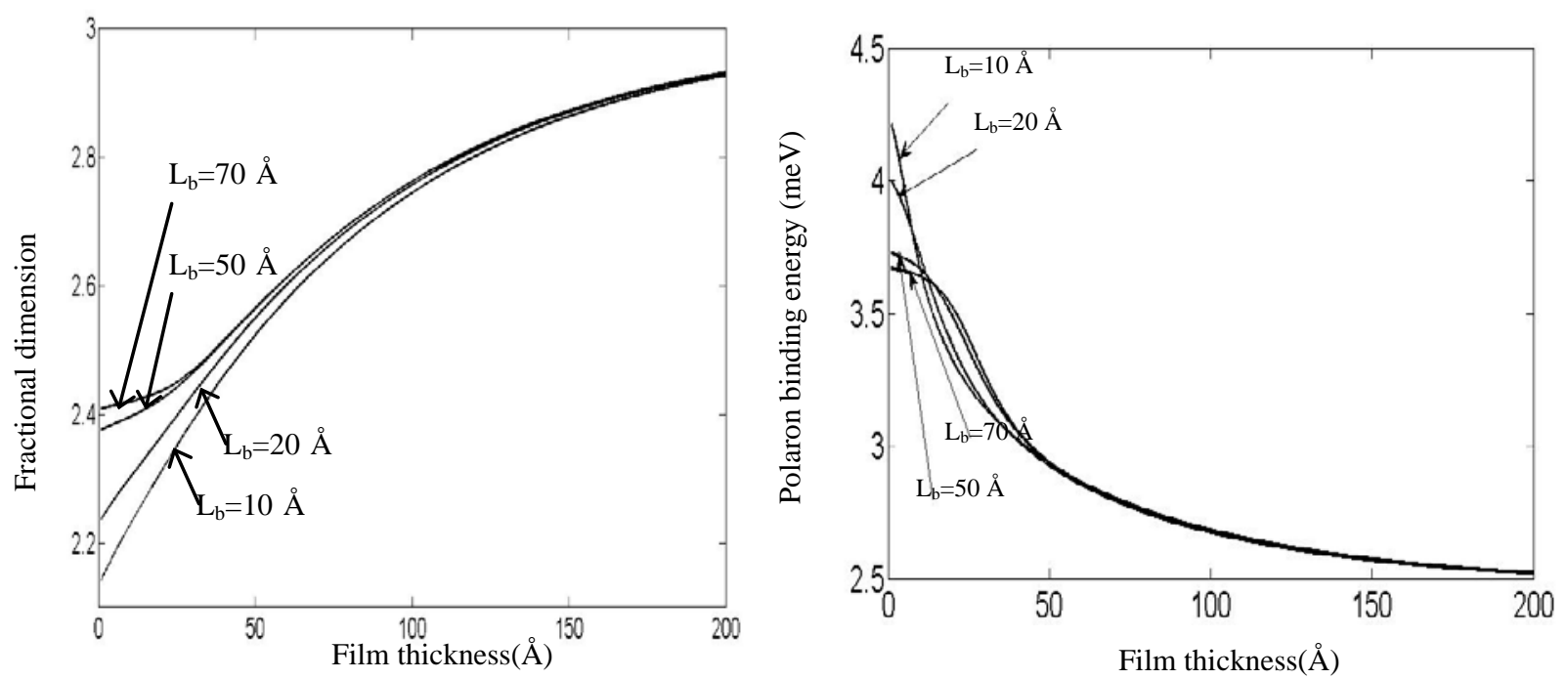

Fig. 1 The fractional dimension as a function of the film thickness for a polaron confined in a GaAs film deposited on the Al0.3Ga0.7As substrate for the substrate thickness $\mathrm{Lb}=10,20,50,70 \AA$.

Fig. 2 The polaron binding energy as a function of the film thickness for a polaron confined in a GaAs film deposited on the Al0.3Ga0.7As substrate for the substrate thickness $\mathrm{Lb}=10,20,50,70$ Å.

\section{Numerical results and discussion}

In order to further investigations of the polaron effect in a GaAs film deposited on $\mathrm{Al}_{0.3} \mathrm{Ga}_{0.7} \mathrm{As}$ substrate, we calculate the binding energy and the effective mass of the polaron. The fractional-dimensional parameter as a function of the film thickness for different substrate thickness $L_{\mathrm{b}}=10,20,50,70 \AA$ is plotted in Fig. 1 . It is seen that the fractional-dimensional parameter starts from different value for different values of substrate thickness, and increases monotonously with increasing the film thickness. As the film thickness increases, the confinement becomes more and more weak, leading to an increase in the fractional dimension that has the same value. It is worth remarking that the different of the fractional dimension become small for the substrate thickness $L_{\mathrm{b}}=50,70 \AA$.

The polaron binding energy and effective mass as a function of the film thickness corresponding to the fraction dimensional parameter results in Fig. 1 is plotted in Fig. 2 and Fig. 3, respectively. From Fig. 2 one can see that for the case of different values of the substrate thickness, the polaron binding energy decreases monotonously with increasing the film thickness. It is worth remarking that, the different values of substrate thickness influence obviously the polaron binding energy for the narrow and medium film thickness, but has no significant influence for very large film thickness. At the same time, one can see that the polaron binding energy is larger for smaller substrate thickness. Notice that this behavior is different from a polaron in sandwich quantum well structure. Because in film structure considered in this paper, the electron is restricted to move in the space less than $\left(L_{w}+L_{b}\right)$, while in finite barrier quantum well, the electron can spread into the barriers on both sides of the well. By continuing to increase the film thickness, the polaron binding energy of $L_{b}=50$ and $70 \AA$ decreases slowly, and occurs for a larger value than the value of $L_{b}=10$ and $20 \AA$. A structure with a shoulder is obtained for the medium film thickness at the values of substrate thickness $L_{b}=50$ and $70 \AA$, respectively. A simple behavior for the film thickness dependence of the fractional-dimensional polaron effective mass is shown in Fig. 3. The different values of the film thickness influence obviously the polaron binding energy and mass shift for narrow and medium film thicknesses, but has no significant influence for large film thicknesses, too. 




Fig. 3 The effective mass as a function of the film thickness for a polaron confined in a GaAs film deposited on the Al0.3Ga0.7As substrate for the substrate thickness $\mathrm{Lb}=10,20,50,70 \AA$.

\section{Conclusions}

In conclusion, we have performed a quite detailed study of a polaron in the GaAs film deposited on Al0.3Ga0.7As system, within the fractional dimensional approach. In this scheme, the actual anisotropic "polaron + film structure" system is modeled by an effective isotropic environment with a fractional dimension. Based on our previous work, a film thickness dependence of the polaron binding energy and effective mass was obtained for the GaAs film deposited on the Al0.3Ga0.7As substrate for different values of substrate thickness. The polaron binding energy and effective mass are found to be sensitive for narrow and medium film thickness. The shoulder in the polaron binding energy appears as a consequence of the competition between the dimensionality and the material parameters. The results obtained in present paper are very useful for further investigating the polaron properties and the optical properties in film structure and have significant meanings for analyzing experimental results, and for some important optoelectric device designs and applications.

\section{References}

[1] X. F. He: Phys. Rev. B Vol. 42 (1990) p. 11751;

[2] X. F. He: Phys. Rev. B Vol. 43 (1991) p. 2063

[3] H. Mathieu, P. Lefebvre and P. Christol: Phys. Rev. B Vol. 46 (1992) p. 4092.

[4] A. Matos-Abiague, L. E. Oliverira and M. de Dios-Leyva: Phys. Rev. B Vol. 58 (1998) p. 4072

[5]E. M. Lopes, D. F. César, F. Franchello, J. L. Duarte, I. F. L. Dias, E. Laureto, D. C. Elias, M. V.

M. Pereira, P. S. S. Guimarães and A. A. Quivy: J. Lumin. Vol. 144 (2013) p. 98

[6] Q. X. Zhao, B. Monemar, P. O. Holtz, M. Willander, B. O. Fimland and K. Johannessen: Phys. Rev. B Vol. 50 (1994) p. 4476

[7] E. Reyes-Gómez, A. Matos-Abiague, C. A. Perdomo-Leiva, M. de Dios-Leyva and L. E. Oliveira: Phys. Rev. B Vol. 61 (2000) p. 13104

[8] Z. P. Wang and X. X. Liang: solid state commun. Vol. 150 (2010) p. 356

[9] T. F. Ronnow, T. G. Pedersen and B. Partoens: Phys. Rev. B Vol. 85 (2012) p. 045412

[10] A. Matos-Abiague, L. E. Oliveira and M. de Dios-Leyva: Physica B Vol. 296 (2001) p. 342

[11] I. D. Mikhailov, F. J. Betancur, R. A. Escorcia and J. Sierra-Ortega: Phys. Rev. B Vol. 67 (2003) p. 115317

[12] J. Kundrotas, A. Čerškus, S. Ašmontas and G. Valušis: Phys. Rev. B Vol. 72 (2005) p. 235322.

[13] A. Thilagam: Phys. Rev, B Vol. 56 (1997) p. 4665

[14] Z. H. Wu, H. Li, L. X. Yan, B. C. Liu and Q. Tian: Physica B Vol. 410 (2013) p. 28

[15] Z. H. Wu, H. Li, L. X. Yan, B. C. Liu and Q. Tian: Superlatt. and Microstruc. Vol. 55 (2013) p. 16

[16] M. A. Smondyrev, B. Gerlach and M. O. Dzero: Phys. Rev. B Vol. 62 (2000) p. 16692

[17] B C Liu, H Li, L. X. Yan, H Sun and Q. Tian: Acta Phys. Sin Vol. 62 (2014) p. 197302 